\title{
SISTEMA DE CRÉDITOS TRANSFERIBLES Y CARGA DE TRABAJO DE LOS ESTUDIANTES EN LAS UNIVERSIDADES DEL CONSEJO DE RECTORES
}

\section{Introducción}

Hacia fines de los años 90 se puso en marcha el programa de Mejoramiento de la Calidad y Equidad de la Educación Superior (MECESUP), que ha promovido profundos cambios en el sistema de educación superior chileno. Las instituciones pertenecientes al Consejo de Rectores de las Universidades Chilenas (CRUCH) han contado con recursos para enfrentar un conjunto de problemas definidos por el mismo MECESUP como estructurales: diseños curriculares caracterizados por una excesiva duración de las carreras y escasa flexibilidad; escasa consideración de los usuarios finales (estudiantes, egresados y empleadores) al momento de diseñar los currículos; baja colaboración interinstitucional, tanto nacional como internacional; altas tasas de deserción en los primeros años y bajas tasas de titulación en los tiempos de diseño de las carreras y escasa movilidad estudiantil dentro del sistema (MECESUP).

Estos desafíos, en el contexto de una dinámica de masificación y diversificación, comienzan a perfilarse como de primera importancia. Las universidades han asumido la tarea de enfrentarlos a través de un conjunto de proyectos que promueven la innovación en las metodologías docentes y la incorporación de tecnologías de información y comunicación que ponen al estudiante y al logro de sus aprendizajes en el centro del proceso docente. En una segunda etapa, se han desarrollado proyectos de rediseño curricular basado en competencias recogiendo la experiencia y los aportes de egresados y empleadores. Estos últimos se han acompañado con la constitución de redes interinstitucionales que han promovido los programas de rediseño en un marco de armonización curricular interuniversitaria. 
Simultáneamente, se observa cómo en otros sistemas de educación superior se promueven acuerdos internacionales con el objeto de fortalecerlos y enfrentar de mejor modo las oportunidades y riesgos de un mercado universitario cada vez más globalizado. El más reciente -y quizá el de mayor repercusión- es el llamado proceso de Bolonia, iniciado luego de la firma del acuerdo de 31 representantes de sistemas de educación de 29 naciones europeas en la Universidad de Bolonia, en 1999 (Bolonia, 1999). A este acuerdo han seguido otros convenios y proyectos que buscan avanzar en la armonización curricular. Entre sus principales resultados está la adopción de un esquema curricular homogéneo, un sistema docente centrado en el aprendizaje de los estudiantes, una formación basada en resultados de aprendizaje y un único sistema de créditos académicos transferible basado en la carga real de trabajo de los estudiantes (ECTS, 2006). Este último elemento, que puede parecer de rango menor y sólo un tecnicismo, se ha revelado como una pieza clave, no sólo para la promoción del intercambio y el reconocimiento académicos, sino, también, para acompañar los procesos de rediseño curricular.

Un estudio sobre créditos académicos, realizado en 2005 por la Pontificia Universidad Católica de Valparaíso, concluye que de las 44 universidades que participaron en el análisis sólo 29 cuentan con un sistema de créditos (Pontificia Universidad Católica de Valparaíso, 2004); algo más del $50 \%$ basa su sistema de créditos en la carga de trabajo de los estudiantes (todas las horas que el estudiante le dedica efectivamente al estudio de un curso), y un número significativo del otro 50\% de la muestra utiliza un sistema basado en la docencia presencial (o directa). Esta diversidad y a veces ausencia de un sistema de créditos muestra una educación superior con barreras estructurales para articular adecuadamente programas académicos entre los currículos, en vistas a la movilidad estudiantil y a la legibilidad de sus carreras.

Partiendo de esta realidad, las universidades pertenecientes al CRUCH han elaborado un conjunto de proyectos y adoptado acuerdos que promueven la movilidad estudiantil, la armonización curricular 
y la elaboración de un sistema de créditos chileno basado en la carga real de trabajo de los estudiantes.

Este artículo describe los principales resultados del proyecto "Sistema de Créditos Transferibles", desarrollado por las universidades del CRUCH y financiado por el Ministerio de Educación a través de un proyecto MECESUP.

\section{Antecedentes}

En el marco de los procesos de convergencia de nuestro sistema de educación superior con los de países con los cuales se han suscrito convenios de cooperación, el CRUCH ha adoptado un conjunto de acuerdos que han servido de base para modelar este proyecto y sus objetivos

Entre los más destacados están la Declaración de Valparaíso, de abril de 2003, que expresa el compromiso de los rectores de "impulsar la adopción de un sistema de créditos compatible de las universidades chilenas entre sí y con el sistema ECTS, y delimitar los requerimientos reales curriculares que se hacen a los estudiantes de acuerdo con la real disponibilidad de tiempo de éstos". El proyecto del que da cuenta este artículo es la respuesta a este acuerdo, y fue presentado en el concurso de 2004 del programa MECESUP, con la participación de todas las universidades del CRUCH. Uno de sus principales resultados es la elaboración del sistema de créditos basado en la carga de trabajo de los estudiantes y compatible con el sistema European Credit Transfer System (ECTS) ${ }^{1}$.

\section{Medición de la carga de trabajo de los estudiantes}

Tras el acuerdo de que el nuevo Sistema de Créditos Transferibles (SCT) debía basarse en la carga real de trabajo de los estudiantes, se

1 Adoptado en forma paulatina por los países de la Unión Europea. 
decidió realizar un estudio que permitiese acercarse a su estimación y cuyos objetivos se resumen en:

- Determinar la carga de trabajo efectiva del estudiante.

- Determinar las cargas de trabajo relativas de la docencia directa y las tareas personales del estudiante.

Es importante destacar el segundo objetivo, que busca dar cuenta de la relación entre el tiempo que el alumno dedica a la docencia directa y aquel que corresponde a su estudio personal, lectura de textos, elaboración de informes, participación en foros por internet y otros trabajos, sean éstos solicitados o recomendados por los docentes.

En el estudio de la carga académica participaron 15.441 estudiantes de 54 carreras de 25 universidades. Éstos informaron de su trabajo durante el segundo semestre de 2005, mediante dos instrumentos: una bitácora y una ficha. Las carreras escogidas fueron las que participaban en alguna de las redes generadas en el marco de los proyectos MECESUP 2004. Este elemento entregaba un valor adicional al estudio, en cuanto las redes pudieron comparar las cargas de trabajo de las mismas carreras dictadas en dos o más universidades.

La bitácora exigía a los estudiantes la identificación de las asignaturas que cursarían y el registro semanal del tiempo dedicado a un conjunto de actividades establecidas en el mismo instrumento y que se describen en la tabla 1. De las 3.276 bitácoras que se recopilaron se pudo estimar, entre otros aspectos, el número de asignaturas que en promedio cursan los estudiantes de las distintas carreras, el tiempo de dedicación a estas asignaturas y la relación entre docencia directa y trabajo personal, así como la distribución de este estudio personal en el conjunto de distintas actividades establecidas en el instrumento.

Al final del semestre, se recogieron 12.165 fichas que permitieron conocer la percepción de los estudiantes respecto de su dedicación a cada asignatura y la distribución del tiempo entre las 
diversas actividades establecidas. Al igual que con la bitácora, con estos datos se pudo establecer la relación entre docencia directa y trabajo personal de los estudiantes.

Los primeros resultados muestran que los estudiantes cursan entre cinco y ocho asignaturas, que en promedio le dedican un tiempo total de 13 horas semanales a cada una y que, en promedio, trabajan 1,3 horas adicionales por cada hora de clase directa. Se observa también una dedicación horaria creciente a lo largo del semestre, la que alcanza una mayor carga de trabajo estudiantil en el mes de noviembre. Estos primeros antecedentes son lógicos y conocidos por estudiantes, académicos y gestores curriculares.

Un segundo grupo de resultados se asocia con las distintas relaciones entre carga docente directa y las actividades que desarrollan fuera del "horario de clases". Por el primer concepto se entiende las horas que los estudiantes asisten a clases, laboratorios, talleres, actividades clínicas y de terreno, entre otras. Las segundas comprenden el estudio personal, lecturas de textos, uso de internet, redacción de informes, preparación de trabajos, por nombrar algunas.

La tabla 1 muestra la agrupación de los estudiantes según distintas distribuciones de su trabajo académico. Estos tres grupos se ordenaron según la dedicación horaria relativa entre clases teóricas y prácticas (presenciales) y el trabajo personal. La última columna presenta el promedio general reportado en el estudio.

Tabla 1: Agrupación de estudiantes según la distribución del trabajo semanal.

\begin{tabular}{l|c|c|c|c}
\hline Actividad & \multicolumn{4}{|c}{ Distribución del trabajo semanal en porcentaje de tiempo } \\
\hline & I & II & III & Promedio \\
\hline Clases teóricas & 30,6 & 24,5 & 10,0 & 25,8 \\
\hline Clases prácticas & 8,9 & 7,9 & 39,6 & 12,7 \\
\hline Evaluaciones & 5,7 & 5,0 & 2,5 & 5,0 \\
\hline Biblioteca & 5,0 & 5,1 & 3,6 & 4,8 \\
\hline Internet & 7,8 & 5,7 & 7,8 & 7,1 \\
\hline Trabajos & 17,3 & 9,2 & 29,3 & 16,3 \\
\hline Estudio personal & 24,8 & 42,4 & 7,3 & 28,2 \\
\hline TOTAL & 100 & 100 & 100 & 100 \\
\hline
\end{tabular}


El grupo I representa el mayor número de estudiantes y no se asocia con ninguna carrera en particular. Se caracteriza, en términos generales, por una relación entre docencia directa (clases teóricas, prácticas y evaluaciones) y trabajo personal (biblioteca, internet, trabajos y estudio personal) cercano a 1 a 1,2 , muy próximo al promedio general.

El grupo II se caracteriza por una fuerte dedicación al estudio personal y se asocia con carreras tales como Derecho y Contador Auditor.

El grupo III -que presenta una mayor carga horaria de clases prácticas y de elaboración de trabajos solicitados en las asignaturas- se asocia con carreras como Arquitectura, Diseño o Trabajo Social.

Al buscar la relación entre la carga de trabajo total y la docencia directa, se observan valores distintos para cada uno de estos grupos, algunos que caen en el rango 2 a 1 hasta 4,2 a 1, si se considera la relación del trabajo total por cada hora de clases.

Los valores registrados para cada uno de los grupos indican que hay una dispersión importante. En la tabla 2 se visualiza esta afirmación.

Tabla 2: Cuocientes entre trabajo total y docencia directa para algunas carreras.

\begin{tabular}{l|c}
\hline Carrera & Cuociente horas trabajo total/horas docencia directa \\
\hline Arquitectura & 4,2 \\
\hline Derecho & 2,9 \\
\hline Ingeniería Comercial & 2,2 \\
\hline Ingeniería Informática & 2,3 \\
\hline Ingeniería Plan Común & 1,9 \\
\hline Química y Farmacia & 2,6 \\
\hline
\end{tabular}

Las cifras señaladas en la tabla 2 no significan un mayor o menor trabajo total de los estudiantes. Por la naturaleza de los instrumentos utilizados no fue posible determinar con certeza los volúmenes de carga total de los estudiantes de las distintas carreras. Sin embargo, 
este valor puede obtenerse, en una primera aproximación, a partir de los datos de la tabla precedente y el número de horas de docencia directa que establece cada currículo.

En los gráficos 1 a 4 se muestra el tipo de trabajo que realizan los estudiantes dentro del trabajo personal fuera de la sala de clases.

Gráfico 1: Distribución porcentual de la carga de trabajo semanal de los estudiantes de Arquitectura.

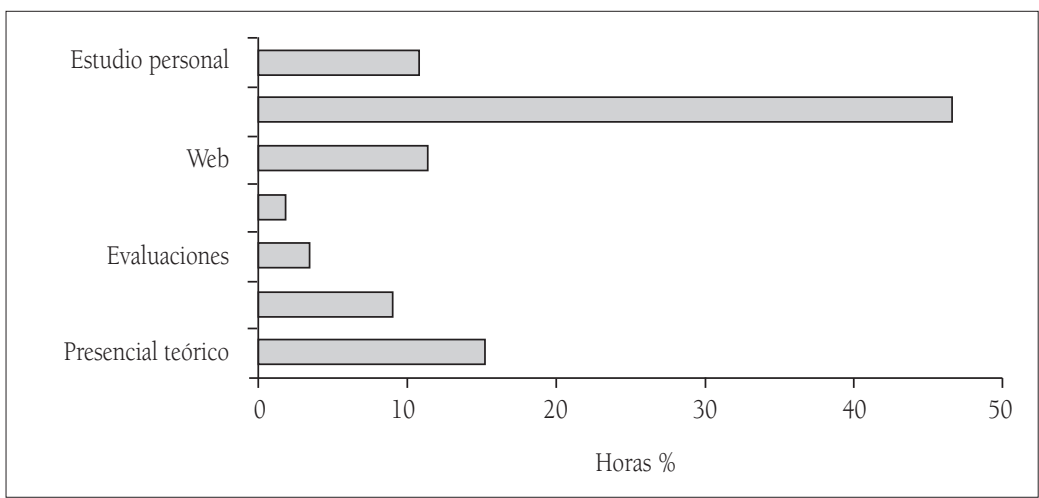

Gráfico 2: Distribución porcentual de la carga de trabajo semanal de los estudiantes de Derecho.

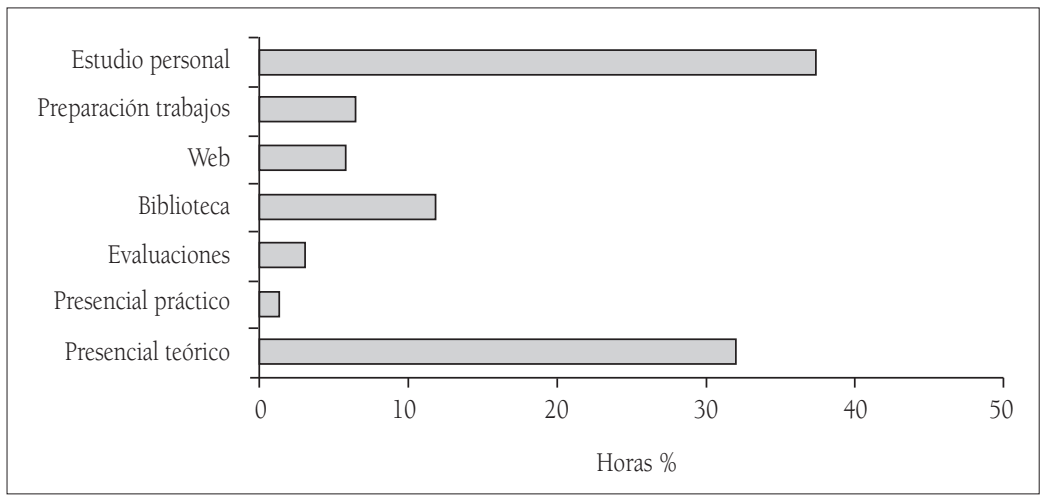


Gráfico 3: Distribución porcentual de la carga de trabajo semanal de los estudiantes de Ingeniería Comercial.

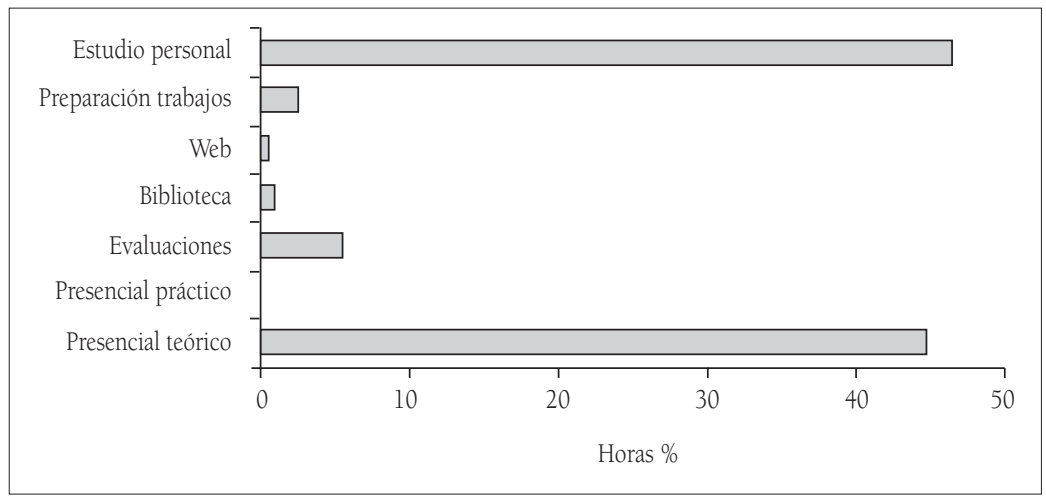

Gráfico 4: Distribución porcentual de la carga de trabajo semanal de los estudiantes de Química y Farmacia.

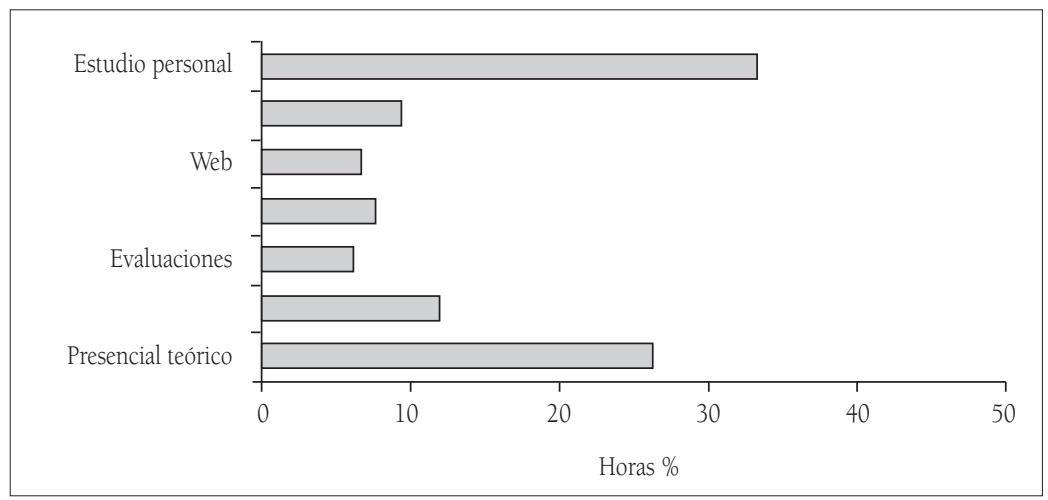

Tal como se observó, los tiempos dedicados a las actividades analizadas presentan un comportamiento heterogéneo, originando grupos con distintas distribuciones de cargas de trabajo. Se constata una variación en la distribución de las cargas de trabajo de las distintas actividades de aprendizaje, dependiendo de las carreras.

Esto lleva a la conclusión más importante de este estudio: la asignación de créditos a una asignatura según la carga de trabajo que demanda no puede ni debe hacerse exclusivamente sobre la base de las 
horas de docencia directa, ya que, como muestran los resultados, una hora de clases para un estudiante de Arquitectura representa un trabajo total muy distinto que para un estudiante de Ingeniería Comercial o de Química y Farmacia o de Derecho o Ingeniería. También, al interior de cada carrera se encuentra que las cargas de trabajo total de las distintas asignaturas no responden necesariamente a una relación única con el número de horas de docencia directa.

En una segunda línea de conclusiones, los gráficos representan con gran aproximación las distintas metodologías utilizadas en cada una de las carreras analizadas. Los estudiantes de Arquitectura, por ejemplo, destinan la mayor parte de su tiempo a la elaboración de trabajos y su carga de trabajo de docencia directa se distribuye aproximadamente en un 30\% para clases prácticas y un $70 \%$ de clases teóricas. En el otro extremo encontramos a los estudiantes de Ingeniería Comercial, que distribuyen su tiempo casi en partes iguales entre la docencia directa y el estudio personal. Los estudiantes de Química y Farmacia dedican un tiempo para asistir a clases teóricas y prácticas, que en total llega casi al $40 \%$ de su carga total, mientras que su trabajo fuera del aula se reparte entre la preparación de trabajos, uso de internet y la biblioteca. En los estudiantes de Derecho el uso de la biblioteca cobra mayor relevancia, el que, sumado al estudio personal, alcanza a casi el 50\% del tiempo total. Por su parte, los estudiantes de Ingeniería Informática destinan casi el $45 \%$ de su tiempo a asistir a clases y su trabajo fuera del aula se distribuye en estudio personal, preparación de trabajos, internet y biblioteca. Estos resultados son de gran utilidad para que los académicos que han diseñado e implementado nuevas estrategias docentes verifiquen el grado de apropiación de éstas por parte de los estudiantes.

La naturaleza de los instrumentos utilizados no permitió obtener de manera directa la carga de trabajo total de los estudiantes en las distintas carreras analizadas. Tampoco se pudo correlacionar el tiempo dedicado a la asignatura con los resultados académicos del estudiante en ella. Con el fin de resolver estas interrogantes y avanzar 
a la instalación de un sistema de monitoreo permanente de la carga de trabajo de los estudiantes, se desarrolló una nueva metodología de estimación de ésta y se aplicó un segundo estudio durante el segundo semestre de 2006, cuyos resultados aún no están disponibles.

\section{De la medición de carga de trabajo de los estudiantes al Sistema de Créditos Transferibles (SCT-Chile)}

En los últimos años se ha avanzado en innovaciones metodológicas y curriculares que han considerado, como elementos centrales, focalizar la docencia en el aprendizaje del estudiante, promover el autoaprendizaje, generar competencias, medir resultados de aprendizaje, mejorar la empleabilidad y darle coherencia al plan de estudios para alcanzar el perfil profesional. En este contexto, la adopción de un sistema común de créditos para las instituciones del CRUCH facilita significativamente la legibilidad de los diversos programas de estudios, ofreciendo a los estudiantes una mejor perspectiva de las oportunidades de estudio fuera de sus universidades de origen. De igual manera, ofrece a las entidades mejores herramientas para la formalización de alianzas y redes, y para el reconocimiento de estudios de alumnos de intercambio.

Un sistema de créditos permite a los académicos, a los gestores curriculares y, en general, a las instituciones, medir, racionalizar y distribuir el trabajo académico de los estudiantes entre las diversas actividades de aprendizaje que componen su plan de estudios. Tal como ha quedado reflejado en el estudio descrito precedentemente, las asignaturas demandan tiempos variables, que no se correlacionan necesariamente con el tiempo de docencia directa.

Por tanto, es un imperativo práctico y ético que las instituciones de educación superior definan de manera clara cómo los componentes de un plan de estudios aportan a la formación establecida en el perfil de egreso, según su propio sello institucional. Esto significa que cada parte juega un papel específico y demanda un trabajo medianamente definido para el estudiante, de modo de hacer exigencias razonables 
para el logro de los objetivos de aprendizaje. Lo razonable se refiere a que deben desarrollarse en el tiempo de diseño de la carrera, desagregado en semestres, semanas por semestre y horas de trabajo por semana.

En términos generales, un sistema de créditos es una forma sistemática de describir un programa de formación, asignando créditos a sus componentes. La definición de los créditos en educación superior puede basarse en distintos parámetros, tales como la carga de trabajo del estudiante, los resultados del aprendizaje y las horas de contacto (ECTS, 2006; De Lavigne, 2003)².

\section{El Sistema de Créditos Transferibles de las universidades del CRUCH}

Se desagrega en tres componentes y está basado en seis principios que permiten orientar su implementación en las diversas instituciones.

El primer componente señala que este sistema se debe basar en la carga de trabajo total de los estudiantes. Esto significa que se debe considerar el tiempo dedicado a cada una de las actividades que debe realizar el alumno para el logro de los objetivos de aprendizaje de cada asignatura: docencia directa, talleres, laboratorios, salidas a terreno, estudio personal, redacción de informes, entre otras.

El segundo dice relación con los tiempos que el estudiante dedica a su carrera. Se asume una carga de trabajo semanal de 45 a 50 horas para una dedicación de tiempo completo en una carrera. De acuerdo con la tabla 3, en la cual se registra el número de semanas más usuales en las universidades chilenas, se obtiene una dedicación total anual en el rango 1.440-1.900 horas.

2 Definición de una unidad de crédito en las universidades de Arizona (2005). Disponible en http://www.abor.asu.edu/1_the_regents/policymanual/chap6/6-601.pdf Último acceso en junio de 2007. 
Tabla 3: Horas de trabajo anual total según número de semanas académicas.

\begin{tabular}{c|c|c}
\hline Semanas académicas anuales & \multicolumn{2}{|c}{ Horas cronológicas semanales } \\
\hline & 45 & 50 \\
\hline 32 & 1.440 & 1.600 \\
\hline 34 & 1.530 & 1.700 \\
\hline 36 & 1.620 & 1.800 \\
\hline 38 & 1.710 & 1.900 \\
\hline
\end{tabular}

Finalmente, el tercer componente es un normalizador anual que permite asignar un número específico de créditos a las distintas actividades curriculares de un año cualquiera del plan de estudios. Se ha convenido que a la carga de trabajo anual total, correspondiente al rango 1.440-1.900 horas, se le asocien 60 créditos SCT.

Por otro lado, con el objetivo de orientar la implementación del SCT-Chile, sin imponer una fórmula, se han acordado un conjunto de principios como ejes de este sistema.

1. Sesenta (60) créditos académicos representan el tiempo de dedicación para que un estudiante de tiempo completo logre los resultados de aprendizaje de un año del Plan de Estudios. Este tiempo varía entre 1.440 y 1.900 horas anuales.

2. La asignación de créditos a una actividad curricular se basa en la carga de trabajo del estudiante.

3. Cada actividad curricular tiene asignado un número de créditos, como proporción del total anual, expresado en enteros.

4. El trabajo total del estudiante sólo puede ser medido por aproximación, puesto que el universo estudiantil presenta alta dispersión y los métodos para recoger información tienen baja confiabilidad.

5. No importando el plan de estudios en que se encuentre, una actividad curricular tiene un único valor en créditos al interior de una institución.

6. La obtención de créditos por parte de un estudiante supone una evaluación y haber superado los mínimos establecidos. 
En general, como consecuencia del principio 1, un año académico de estudios de tiempo completo equivale a 60 créditos, un semestre a 30 créditos y un trimestre a 20 créditos. De este modo, un programa de estudios de cuatro años corresponderá a 240 créditos, uno de cinco a 300 y uno de siete años a 420 créditos. Por su parte, un (1) crédito SCT equivale a una carga de trabajo en el rango de 24 a 31 horas.

La siguiente fase dentro de este proceso modernizador del sistema chileno será la implementación del SCT al interior de cada una de las universidades. Para ello se contará con el apoyo del programa MECESUP, a través de un proyecto que permitirá la instalación de una red de personal especializado para apoyar los procesos de innovación curricular y de movilidad estudiantil. En este marco, se encuentra en elaboración una guía práctica $(\mathrm{CRUCH}, 2007)$ que ayude a la instalación del sistema de créditos en las instituciones que decidan su implementación.

Implementar un nuevo sistema de créditos basado en la carga de trabajo de los estudiantes se ha revelado como una actividad de gran riqueza institucional. Por ejemplo, dado que los 60 créditos anuales se deben "repartir" entre las diversas actividades curriculares, se asocia con cada actividad un número de horas que "le pertenecen"; o, visto de otra forma, cada actividad "consume" un cierto número de horas de un máximo total establecido. Entonces, en un sistema en que "compiten", por ejemplo, las ciencias básicas, la formación general, la formación disciplinaria, los idiomas y el deporte, entre otros, se pone en tensión a los actores involucrados. Por esta razón, la implementación del SCT-Chile es un proceso que exige considerar la cultura institucional, demandando negociaciones a la hora de asignar créditos a las diversas actividades curriculares y poniendo de relieve las complementarias y optativas.

Finalmente, las universidades del $\mathrm{CRUCH}$ han asumido el acuerdo de implementar un SCT como un elemento de trabajo para ser contextualizado en el marco de una política más amplia de renovación 
curricular, constituyéndose también en una pieza clave que posibilite el diálogo interinstitucional y la movilidad de los diferentes actores dentro y fuera del sistema.

\section{Agradecimientos}

Al programa MECESUP, por el financiamiento del proyecto que sustenta este estudio, y a los integrantes del Comité Directivo del proyecto SCT: Iñigo Díaz, Juan José Ugarte, Enrique Montenegro, Ernesto Figueroa, Patricio Jiménez y Juan Oyarzo. También a Roxana Pey, Claudia Reyes y Marcelo López, del programa MECESUP, por su apoyo y coordinación, y a Sara Chauriye e Iván Atencio, por sus aportes en el desarrollo del proyecto y colaboración en los aspectos metodológicos.

\section{Referencias bibliográficas}

CRUCH. (2007) Guía Práctica SCT-Chile (versión preliminar).

De Lavigne, Richard. (2003) Créditos ECTS y Métodos para su Asignación. Disponible en http://www.aneca.es/modal_eval/docs/doc_conv_grall. pdf Último acceso en junio de 2007.

Declaración de Bolonia para la Constitución del Espacio Europeo de Educación Superior. (1999) Disponible en http://ec.europa.eu/education/policies/ educ/bologna/bologna.pdf Último acceso en junio de 2007.

ECTS. (2006) Sistema Europeo de Transferencia y Acumulación de Créditos. Disponible en http://ec.europa.eu/education/programmes/socrates/ ects/index_es.html Último acceso en junio de 2007.

MECESUP. Políticas y Objetivos. Disponible en http://www.mecesup.cl/proy_ mece2/politic.html Último acceso en junio de 2007.

Pontificia Universidad Católica de Valparaíso. (2004) Sistema de Creditaje en Universidades Nacionales. Valparaíso: PUCV.

Recibido: 22 de mayo de 2007

Aceptado: 11 de junio de 2007 